\title{
Capital Inflows and Silver Standard in
}

India

\section{1}

Dr. Debesh Bhowmik

Retired Principal

International Institute for Development Studies,

Kolkata

\section{Abstract}

In this paper author tries to relate gold and silver inflows with GDP, GDP per capita, export, import and gold silver price ratio in India during silver standard regime from 1851 to 1893. Author used semi-log, double-log regression models, Johansen co-integration and VAR models (1991,1996) and Bai-Perron model (2003) for structural change taking data from Maddison(2006) and Ambedkar(1923). The paper concludes that gold inflows during 18511893 had decreased at the rate of $0.34 \%$ per year insignificantly but it was nonstationary, convergent and had no structural breaks. Silver inflows during 1851-1893 had increased at the rate of $1.51 \%$ per year insignificantly and found nonstationary and convergent and had one upward structural break in 1857. No cointegration among gold or silver inflows with GDP, GDP per capita, export, import and gold silver price ratio was found during 1851-1893 where VAR model was unstable and non-stationary and impulse response functions were diverging. Semi-log linear regression model among silver inflows and gold inflows with those variables were also insignificant although GDP, export, import and gold silver price ratio had been increasing at the rates of $0.52 \%, 9.14 \%, 5.16 \%$ and $0.77 \%$ per year significantly. But doublelog linear regression model suggested that gold inflows had significant impact from GDP, GDP per capita, export, and gold-silver price ratio but had no significant impact of silver inflows from those variables during 1851-1893 respectively. Yet, there is bidirectional causality among gold inflows, GDP, GDP per capita, export, import and gold silver price ratio significantly during the given period.Even, there were sharp depreciation of rupee sterling rate, falling silver price, silver production and rising gold price and gold production during the silver standard regime. Thus, gold and silver inflows could not synthesize the silver standard more effective in macro-dynamic adjustment during 1851-1893 although the series of managerial experiments of the commissions and government are equally responsible for instability of the silver standard in India which was equally identical with gold standard in England

Key words-Net gold inflows, net silver inflows,silver standard,GDP,export, import,co-integration, VAR

JEL-E42, F33, N10, N20

\section{Introduction}

Silver standard in India was introduced in 1835 but the Act of XVII and the Act of XXI in 1835 declared both silver coin and copper coins as legal tender, on the other hand, gold coin was not legal tender yet it was circulated. Later on, in 1861 by Act of XIX, gold coin was treated as legal tender.In 1861 ,the paper currency notes were circulated.The gold: silver was 1:15.5 and rupee sterling rate was fixed at $1 \mathrm{~s} 10.5 \mathrm{~d}$ where exchanges were governed by relative values of gold and silver.

During long 400 years from 1493 to 1893 ,gold and silver production were more or less uniform but during 1600-1700,index of gold rose from 
130 to 176 , which rose to 270 during 1700 $1800 . \operatorname{In} 1870$, the index of gold production stipulated to 2124 as compared to 450 for silver.Even the rupee sterling rate depreciated and price of gold silver ratio appreciated to a lager extend.India was one of chief producer of the silver and gold but it was the net importerof both gold and silver which were volatile. Although silver standard during 1873-1893 in India was as like as gold standard in England during 18731893,yet British government introduced several policies of mints, currency circulation as well as bimetallism as an experimental basis which made the silver standard unstable. During this period, most of the countries in the world started to introduce gold standard including British colonies. In India, gold supplies and its prices were stipulating compared to silver,but British Government denied to introduce gold standard in spite of numerous positive signals of implementing gold standard given by many commissions.In 1893, England declared gold exchange standard in India where gold was not convertible to rupee but rupee was convertible to sterling which was fixed parity with gold. Therefore,success story of silver standard is little yet there is no vital disturbance in working the system of silver standard in India.

\section{Objective of the paper}

In this paper author endeavors to analysis the working of capital inflows in the silver standard in India and its relation with the GDP ,GDP per capita and on international trade and even on the gold silver price ratio during 1851-1893.The net gold import and net silver import were considered as capital flows for the specified period.

\section{Methodology and data}

Net gold import and net silver import were treated as capital flows in India during 1851-1893. The trend lines of gold inflows,silver inflows, export, import, GDP, GDP per capital, ratio of gold and silver price were calculated by semi-log linear
model.Stationary was observed through ARIMA model, structural change was shown by BaiPerron model (2003).Double-log multiple regression model was used for showing relationship among those variables with gold and silver inflows for the specified period. Since there is no co-integration with gold inflows and other variables and silver inflows with other variables,author used Johansen VAR model $(1991,1996)$ for showing relationship analyzing residual tests and impulse response functions. Even,Granger (1969) model was tested for causality.Data for GDP and per capita GDP were collected from Maddison(2006) and data for all other variables were taken from B.R.Ambedkar(1923). Assume, $\mathrm{x}_{1}=\mathrm{GDP}, \mathrm{x}_{2}=\mathrm{GDP}$ per capita, $\mathrm{X}_{3}=$ export, $\mathrm{x}_{4}=$ import, $\mathrm{x}_{5}=$ gold silver price ratio, $\mathrm{y}_{1}=$ net gold import, $\mathrm{y}_{2}=$ net silver import

\section{Literature Review}

Davis and Gallman(2001) analyzed that real GDP and per capita GDP growth had fallen from 1861-1889 to 1889-1904 in Australia due to declining contribution of gold and silver.Bordo and Meissner(2007) verified that capital flows increases growth rate in most of the countries during 1880-1913.Bai and Kung(2016) explained through ARDL approach that a decrease in global supply of precious metal had together culminated in the outflow of silver from China depressing inflation, depreciating value of copper relative to silver diminishes standard of living. Gonzalez, Galvarriato and Williamson (2008) verified that Mexican GDP doubled due to increasing international trade, silver export, and increase in textiles during 1796-1872. Gault(2014) showed using world bank monthly data from 1970 to 2011 that world trade rose due to rise in gold but gold price did not relate with GDP growth. In international monetary system, gold is a proxy for some macro parameters.Bordo(1981)showed that gold flows in USA led to increase in money supply which induced to rise inflation rate and increased 
imports and external deficit where he verified that coefficient of variation of per capita income in USA and UK increased from 3.5\% and $2.5 \%$ during $1870-1913$ to $5.5 \%$ and $4.9 \%$ during $1919-1938$ which again fell to $1.6 \%$ and $1.4 \%$ during 1946-1979 respectively as a result of gold inflows.SRSrocco report(2015) asserted that during 1970-1990, price of oil increased 16 times its 1971 level,silver shot up 16 times and gold jumped 15 times after collapse of gold dollar convertibility and the same was happened during 2000-2012. Expert opinion said that it was due to a fundamental change in energy market not a change in bull market.World gold council(2010) showed that gold price development do not resembles past bubble including US housing price,NASDAQ, Nikkei equity market bubble.Gold price is consistent with long range average assets including oil.Gold demand becomes robust due to emerging markets,shift of behavior of central banks, recovery and new advance in industrial demand for gold.O'Connor et al(2015) showed some important findings.Over 15 years in UK and USA,real interest rate decreased as a result of increase in real price of gold.In USA during 1985-2015,there was no co-integration between gold and CPI but during 1973-1983,gold price is positively related with US inflation which was true also during 1945-1983.In Euro Area during 1969-2011,gold is found to be a partial hedge for inflation.It was learnt from a PhD student of Japan that Japan was in silver standard before 1897.During 1885-1897, silver fell down and depreciation of silver led to increase in export and increase in GDP and GDP per capita.A 3.5\% increase in export led to one percent increase in GDP was seen.

\section{Some observations of the model}

During the silver standard regime in India from 1851 to 1893 , net gold inflows had been decreasing at the 0.34 per cent per year which was insignificant. $\log \left(y_{1}\right)=14.770-0.003433 t$

$$
(58.54) *(-0.34)
$$

$R^{2}=0.0028, \mathrm{~F}=0.118, \mathrm{DW}=0.46, y^{2}=$ net gold inflows(imports), $\quad *=$ significant at $5 \%$ level.t=year

Net gold inflow from 1851 to 1893 is convergent but nonstationary because its $A R(1)$ is convergent and stationary but its $M A(1)$ is convergent and nonstationary.

$\log \left(\mathrm{y}_{\mathrm{t} t \mathrm{t}}\right)=14.63648+0.7232 \log \left(\mathrm{y}_{\mathrm{t}-1}\right)+\varepsilon_{\mathrm{t}}+0.083569 \varepsilon_{\mathrm{t}-1}+0.26009 \sigma^{2}$

$$
(40.19)^{*}(3.46)^{*}
$$

$R^{2}=0.58, \mathrm{~F}=18.57, \mathrm{DW}=1.97$, inverted $\mathrm{AR}$ root $=0.72$, inverted MA root $=-0.08$ ,${ }^{*}=$ significant at $5 \%$ level.

This series has no structural breaks during the period.

On the other hand, net inflow of silver in India during silver standard from 1851 to 1893 had been stipulating at the rate of $1.51 \%$ per year which was insignificant.

$\log \left(y_{2}\right)=15.034+0.015138 t$

$$
(43.84) *(1.115)
$$

$R^{2}=0.029, \mathrm{~F}=1.24, \mathrm{DW}=1.36, \mathrm{y} 2=$ net inflow of silver, ${ }^{*}=$ significant at $5 \%$.

In Fig-1,the upward fitted line is shown clearly.

Fig-1:Trend line of silver inflows

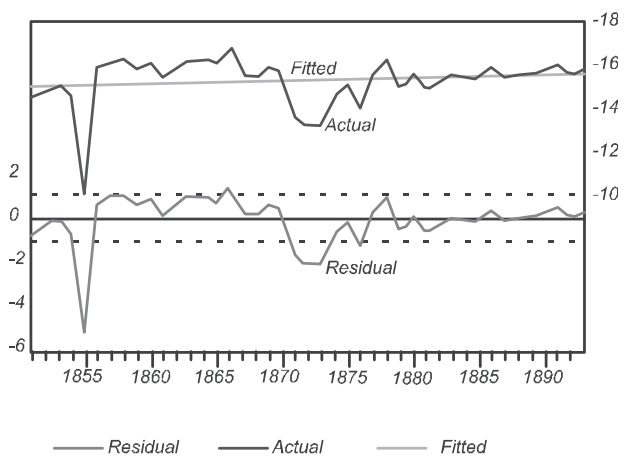

Source-Computed by author 
The net inflow of silver in India during 1851-1893 is non-stationary but convergent which is shown by ARIMA $(1,1,1)$ model. It is not a good fit yet it is stable.

$\log \left(\mathrm{y}_{2 \mathrm{t}}\right)=15.363+0.48873 \log \left(\mathrm{y}_{2 \mathrm{t}-1}\right)+\varepsilon_{\mathrm{t}}-0.183616 \varepsilon_{\mathrm{t}-1}+1.0639 \sigma^{2}$

$$
(35.71)^{*}(0.96) \quad(-0.34) \quad(7.32)^{*}
$$

$R^{2}=0.11, \mathrm{~F}=1.64, \mathrm{DW}=1.96$, inverted $\mathrm{AR}$ root $=0.49$, inverted $\mathrm{MA}$ root $=0.18,{ }^{*}=$ significant at $5 \%$ level.

Net inflow of silver has one upward structural breaks in 1857 only. This is verified by Bai-Perron test(2003)in which HAC standard errors and covariance was assumed and trimming 0.15 with maximum 5 beaks is assumed.

Table-1: Structural breaks of net inflow of silver

\begin{tabular}{|l|l|l|l|l|}
\hline variables & coefficient & Standard error & T statistic & Probability \\
\hline & \multicolumn{2}{|l|}{$1851-1856=60 \mathrm{obs}$} & & \\
\hline c & 14.2328 & 0.3368 & 42.25 & 0.00 \\
\hline & $1857-1893=37$ obs & & \\
\hline c & 15.537 & 0.218 & 71.30 & 0.00 \\
\hline
\end{tabular}

$R^{2}=0.17, \mathrm{~F}=8.64^{*}, \mathrm{DW}=1.62$; Source-Computed by author

In Fig-2,the upward structural break in 1857 is shown clearly.

Fig-2:Structural break

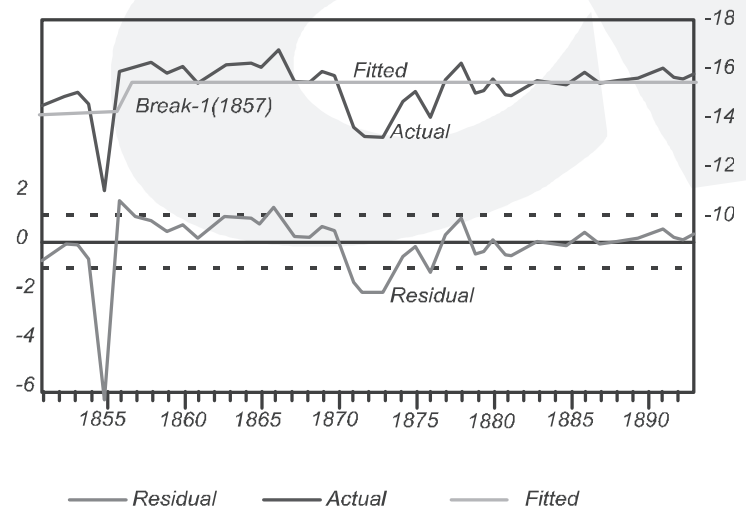

Source-Computed by author

Double log multivariate regression model showed that one per cent increase in GDP,GDP per capita,export,import,gold silver price ratio and net silver inflow led to $12.68 \%$ decrease, $19.27 \%$ increase, $1.89 \%$ increase, $1.47 \%$ increase, $9.93 \%$ decrease and $0.13 \%$ increase in net inflows of gold per year respectively where relation between gold inflows and GDP,GDP per capita, export, goldsilver price ratio are significant at $5 \%$ level. 
$\log \left(\mathrm{y}_{1}\right)=-19.359-12.683 \log \left(\mathrm{x}_{1}\right)+19.275 \log \left(\mathrm{x}_{2}\right)+1.89 \log \left(\mathrm{x}_{3}\right)+1.47 \log \left(\mathrm{x}_{4}\right)-9.938 \log \left(\mathrm{x}_{5}\right)+0.1319 \log \left(\mathrm{y}_{2}\right)$
$(-0.56)(-1.99)^{*}$
$(2.86)^{*}$
$(2.54)^{*}$
(1.53)
$(-3.35)^{*}$

$R^{2}=0.48, \mathrm{~F}=5.67^{*}, \mathrm{DW}=1.24$, where $\mathrm{x}_{1}=\mathrm{GDP}, \mathrm{x}_{2}=\mathrm{GDP}$ per capita, $\mathrm{x}_{3}=$ export, $\mathrm{x}_{4}=\mathrm{import}, \mathrm{x}_{5}=$ gold silver price ratio, $\mathrm{y}_{2}=$ net inflows of silver,${ }^{*}=$ significant at $5 \%$ level

Similarly,one per cent increase in GDP, GDP per capita, export, import, gold silver price ratio and net gold inflow per year led to $11.11 \%$ fall, $12.13 \%$ rise, $1.86 \%$ increase, $0.045 \%$ rise, $1.47 \%$ increase and $0.37 \%$ increase in net silver inflows in India per year during 1851-1893 in silver standard regime which are all insignificant.

$\log \left(\mathrm{y}_{2}\right)=2.739-11.1162 \log \left(\mathrm{x}_{1}\right)+12.139 \log \left(\mathrm{x}_{2}\right)+1.86 \log \left(\mathrm{x}_{3}\right)-0.045 \log \left(\mathrm{x}_{4}\right)+1.47 \log \left(\mathrm{x}_{5}\right)+0.37 \log \left(\mathrm{y}_{1}\right)$
$(0.047)(-1.10)$
$(0.98)$
$(1.416) \quad(-0.027)$
$(0.25)$

(1.35)

$R^{2}=0.24, \mathrm{~F}=1.89, \mathrm{DW}=1.59$,

To show linear combination of silver inflows with other variables, Johansen Co-integration test suggests that there are no co-integrating vectors shown by Trace and Max Eigen Statistic (Table-2).

Table-2:Co-integration test

\begin{tabular}{|l|l|l|l|l|}
\hline Hypothesized no. of CEs & Eigen value & Trace statistic & $0.05 \mathrm{CV}$ & Probability* \\
\hline None & 0.524 & 113.692 & 125.615 & 0.211 \\
\hline At most 1 & 0.445 & 83.189 & 95.753 & 0.266 \\
\hline At most2 & 0.412 & 58.993 & 69.818 & 0.267 \\
\hline At most3 & 0.299 & 37.219 & 47.856 & 0.337 \\
\hline At most4 & 0.245 & 22.630 & 29.797 & 0.264 \\
\hline At most5 & 0.236 & 11.095 & 15.494 & 0.205 \\
\hline At most6 & 0.0006 & 0.026 & 3.841 & 0.87 \\
\hline Hypothesized no of CEs & Eigen value & Max Eigen statistic & 0.05 CV & Probability* \\
\hline None & 0.524 & 30.502 & 46.231 & 0.75 \\
\hline At most 1 & 0.445 & 24.196 & 40.077 & 0.825 \\
\hline At most2 & 0.412 & 21.774 & 33.876 & 0.625 \\
\hline At most3 & 0.299 & 14.588 & 27.584 & 0.779 \\
\hline At most4 & 0.245 & 11.534 & 21.131 & 0.593 \\
\hline At most5 & 0.236 & 11.068 & 14.264 & 0.150 \\
\hline At most6 & 0.0006 & 0.0266 & 3.841 & 0.870 \\
\hline
\end{tabular}

*=MackinnonHaugMichelis(1999) p values 
Since there is no co-integration ,The estimated VAR model is given below.

$\Delta \mathrm{x}_{1 \mathrm{t}}=8451.51+0.212 \Delta \mathrm{x}_{1 \mathrm{t}-1}-2.161 \Delta \mathrm{x}_{2 \mathrm{t}-1}+3.10 \mathrm{E}-06 \Delta \mathrm{x}_{3 \mathrm{t}-1}+1.53 \mathrm{E}-05 \Delta \mathrm{x}_{4 \mathrm{t}-1}+174.27 \Delta \mathrm{x}_{5 \mathrm{t}-1}-9.07 \mathrm{E}-06 \Delta \mathrm{y}_{1 \mathrm{t}-1}+7.32 \mathrm{E}-06 \Delta \mathrm{y}_{2 \mathrm{t}-1}$
$(3.6)$
(1.02)
$(-0.54)$
$(0.32)$
(1.27)
(1.45)
$(-0.22)$
$(0.51)$

$R^{2}=0.92, F=56.29, A I C=14.47 \quad S C=14.80$

$\Delta \mathrm{x}_{2 \mathrm{t}}=313.711-0.025 \Delta \mathrm{x}_{1 \mathrm{t}-1}+0.796 \Delta \mathrm{x}_{2 \mathrm{t}-1}+1.20 \mathrm{E}-07 \Delta \mathrm{x}_{3 \mathrm{t}-1}+3.32 \mathrm{E}-07 \Delta \mathrm{x}_{4 \mathrm{t}-1}+7.67 \Delta \mathrm{x}_{5 \mathrm{t}-1}-8.67 \mathrm{E}-07 \Delta \mathrm{y}_{1 \mathrm{t}-1}+2.37 \mathrm{E}-07 \Delta \mathrm{y}_{2 \mathrm{t}-2}$
$(3.97)^{*}(-3.53)^{*}$
$(5.83) *(0.365)$
$(0.807)$
(1.86)
$(-0.86)$
(0.48)

$\mathrm{R}^{2}=0.765, \mathrm{~F}=15.81, \mathrm{AIC}=7.72, \mathrm{SC}=8.05$

$\Delta \mathrm{x}_{3 \mathrm{t}}=-46481278+4953.34 \Delta \mathrm{x}_{1 \mathrm{t}-1}-137563.9 \Delta \mathrm{x}_{2 \mathrm{t}-1}+0.466 \Delta \mathrm{x}_{3 \mathrm{t}-1}+0.068 \Delta \mathrm{x}_{4 \mathrm{t}-1}+4792340 \Delta \mathrm{x}_{5 \mathrm{t}-1}+1.919 \Delta \mathrm{y}_{1 \mathrm{t}-1}-0.355 \Delta \mathrm{y}_{2 \mathrm{t}-1}$
$(-1.08)$
$(1.27)$
$(-1.84)$
$(2.59)^{*}$
$(0.305)$
$(2.14)^{*}$
$(2.57)^{*}$
$(-1.33)$

$\mathrm{R}^{2}=0.96, \mathrm{~F}=130.0^{*}, \mathrm{AIC}=34.14, \mathrm{SC}=74.47$

$\Delta \mathrm{x}_{4 \mathrm{t}}=-1.11 \mathrm{E}+08+9232.72 \Delta \mathrm{x}_{1 \mathrm{t}-1}-84374.41 \Delta \mathrm{x}_{2 \mathrm{t}-1}+0.084 \Delta \mathrm{x}_{3 \mathrm{t}-1}+0.3377 \Delta \mathrm{x}_{4 \mathrm{t}-1}+3264343 \Delta \mathrm{x}_{5 \mathrm{t}-1}+0.2086 \Delta \mathrm{y}_{1 \mathrm{t}-1}-0.235 \Delta \mathrm{y}_{2 \mathrm{t}-1}$
$(-3.92)^{*}(3.61)^{*}$
$(-1.72)$
$(0.713)$
$(2.29)^{*}$
$(2.21)^{*}$
$(0.425)$

$\mathrm{R}^{2}=0.98, \mathrm{~F}=247.81^{*}, \mathrm{AIC}=33.3, \mathrm{SC}=33.63$

$\Delta \mathrm{x}_{5 \mathrm{t}}=4.0918-8.24 \mathrm{E}-05 \Delta \mathrm{x}_{1 \mathrm{t}-1}+0.00176 \Delta \mathrm{x}_{2 \mathrm{t}-1}+4.27 \mathrm{E}-09 \Delta \mathrm{x}_{3 \mathrm{t}-1}-2.07 \mathrm{E}-08 \Delta \mathrm{x}_{4 \mathrm{t}-1}+0.716 \Delta \mathrm{x}_{5 \mathrm{t}-1}-6.34 \mathrm{E}-08 \Delta \mathrm{y}_{1 \mathrm{t}-1}+1.22-$ $\mathrm{E} 08 \Delta \mathrm{y}_{2 \mathrm{t}-1}$
(1.8)
$(-0.401)$
(0.439)
(0.45)
(1.75)
$(6.06)^{*}$
$(-1.73)$
$(0.908)$

$\mathrm{R}^{2}=0.97, \mathrm{~F}=212.09^{*}, \mathrm{AIC}=0.629, \mathrm{SC}=0.96$

$\Delta \mathrm{y}_{1 \mathrm{t}}=-8108862+636.61 \Delta \mathrm{x}_{1 \mathrm{t}-1}+4042.18 \Delta \mathrm{x}_{2 \mathrm{t}-1}-0.0215 \Delta \mathrm{x}_{3 \mathrm{t}-1}-0.00204 \Delta \mathrm{x}_{4 \mathrm{t}-1}-76501.53 \Delta \mathrm{x}_{5 \mathrm{t}-1}+0.8244 \Delta \mathrm{y}_{1 \mathrm{t}-1}+0.033 \Delta \mathrm{y}_{2 \mathrm{t}-1}$
$(-0.81) \quad(0.708)$
$(0.23)$
$(-0.51) \quad(-0.03)$
$(-0.14)$
$(4.78)^{*}$
$(0.54)$

$\mathrm{R}^{2}=0.67, \mathrm{~F}=10.28, \mathrm{AIC}=31.21, \mathrm{SC}=31.54$

$\Delta \mathrm{y}_{2 \mathrm{t}}=-63316662+3366.48 \Delta \mathrm{x}_{1 \mathrm{t}-1}+311.52 \Delta \mathrm{x}_{2 \mathrm{t}-1}-0.034 \Delta \mathrm{x}_{3 \mathrm{t}-1}-0.204 \Delta \mathrm{x}_{4 \mathrm{t}-1}+1705435 \Delta \mathrm{x}_{5 \mathrm{t}-1}+1.036 \Delta \mathrm{y}_{1 \mathrm{t}-1}+0.235 \Delta \mathrm{y}_{2 \mathrm{t}-1}$

$\begin{array}{llllll}(-2.56)^{*} & (0.50) & (0.007) & (-0.33) & (-1.58) & (1.3)\end{array}$

$\mathrm{R}^{2}=0.42, \mathrm{~F}=3.63, \mathrm{AIC}=33.03, \mathrm{SC}=33.36, *=$ significant at $5 \%$ level 
The estimated VAR model states that [i] change of GDP per capita is negatively related with change of previous period's GDP and positively related with previous period's GDP per capita,[ii] change of export is positively related with change of previous period's export, ratio of gold and silver price,change of gold inflows,[iii] change of import is positively related with change of previous period's GDP,import and gold silver price ratio,[iv]change of gold and silver inflows are positively related with their previous period. Other relations are insignificant.

This VAR model is unstable because one of its 7 roots is greater than one ,two roots are imaginary and 4 roots are less than one (,ie 1.012998, $0.853108 \pm 0.084339 i, 0.527198,0.307550$, 0.272081 ), so all roots do not lie inside the unit root circle.It is seen in the Fig-3.
Fig-3:Inverse roots of AR characteristic polynomial

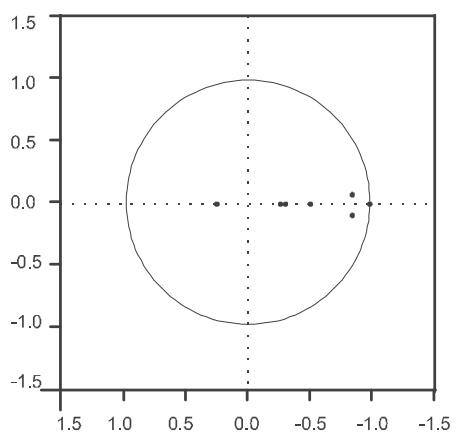

Source-Computed by author

The impulse response functions are diverging so that it is non-stationary and unstable. The exogenous shocks could not tend the model into equilibrium.

Fig-4:Impulse response functions
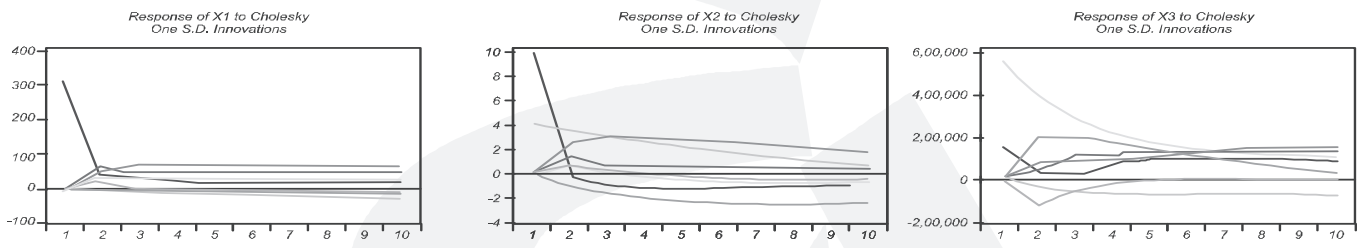

$\begin{array}{rrr}x_{1} & x_{2} & x_{3} \\ x_{4} & -x_{5} & -y_{1}\end{array}$

$\begin{array}{rrr}x_{1} & -x_{2} & x_{3} \\ x_{4} & -x_{5} & -{ }^{1} \\ y_{2} & & \end{array}$

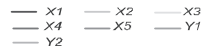

Response of $X 4$ to Cholesky

Response of $X 5$ to Cholesky
One SD Inrovations

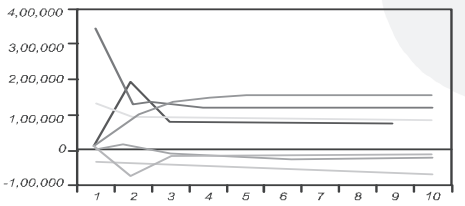

\begin{tabular}{rrr}
$x_{1}$ & $-X_{2}$ & $x_{3}$ \\
\hline$X^{4}$ & $-X_{5}$ & $-Y_{1}$
\end{tabular}

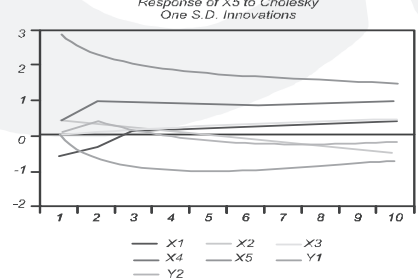

Response of $Y 1$ to Cholesky
One S.D. Innovations
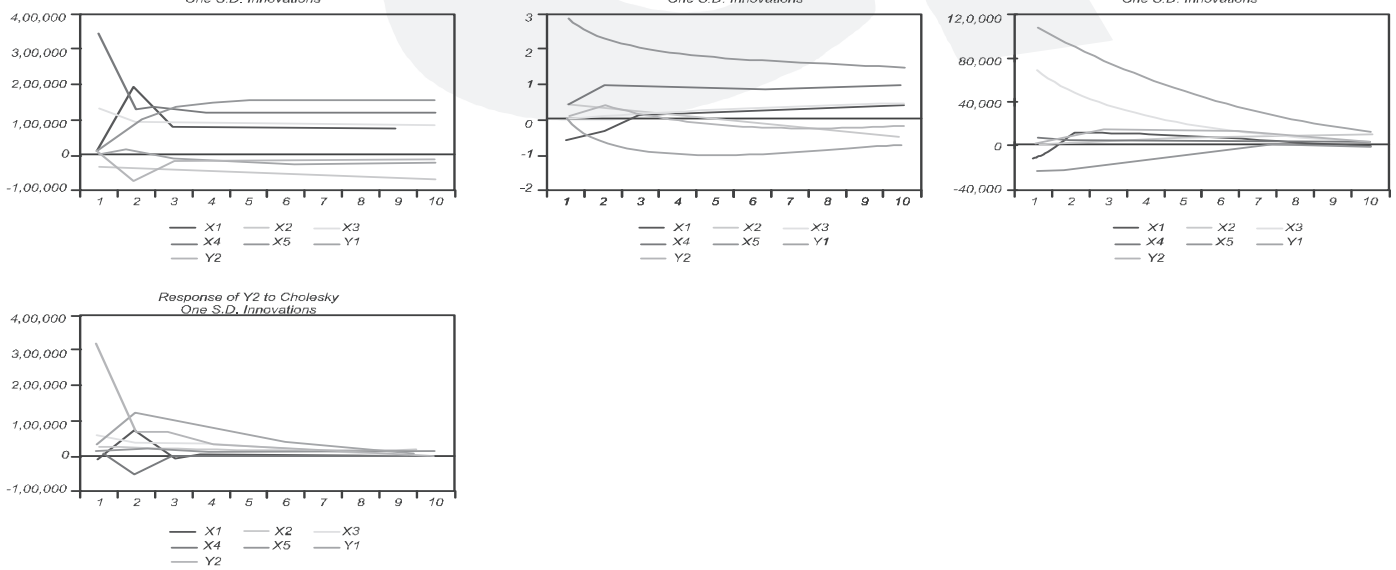

Source-Computed by author 
The residuals test of VAR model assures that the VAR model has problems of autocorrelations which is seen in the Fig-5.

Fig-5:Problem of autocorrelation
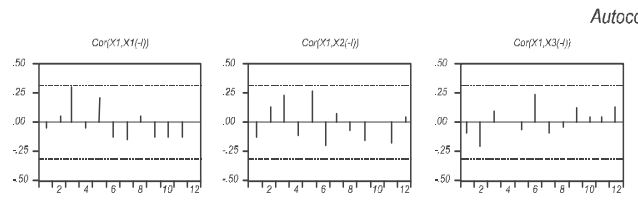

utocorrelations with 2 Std. Err. Bounds
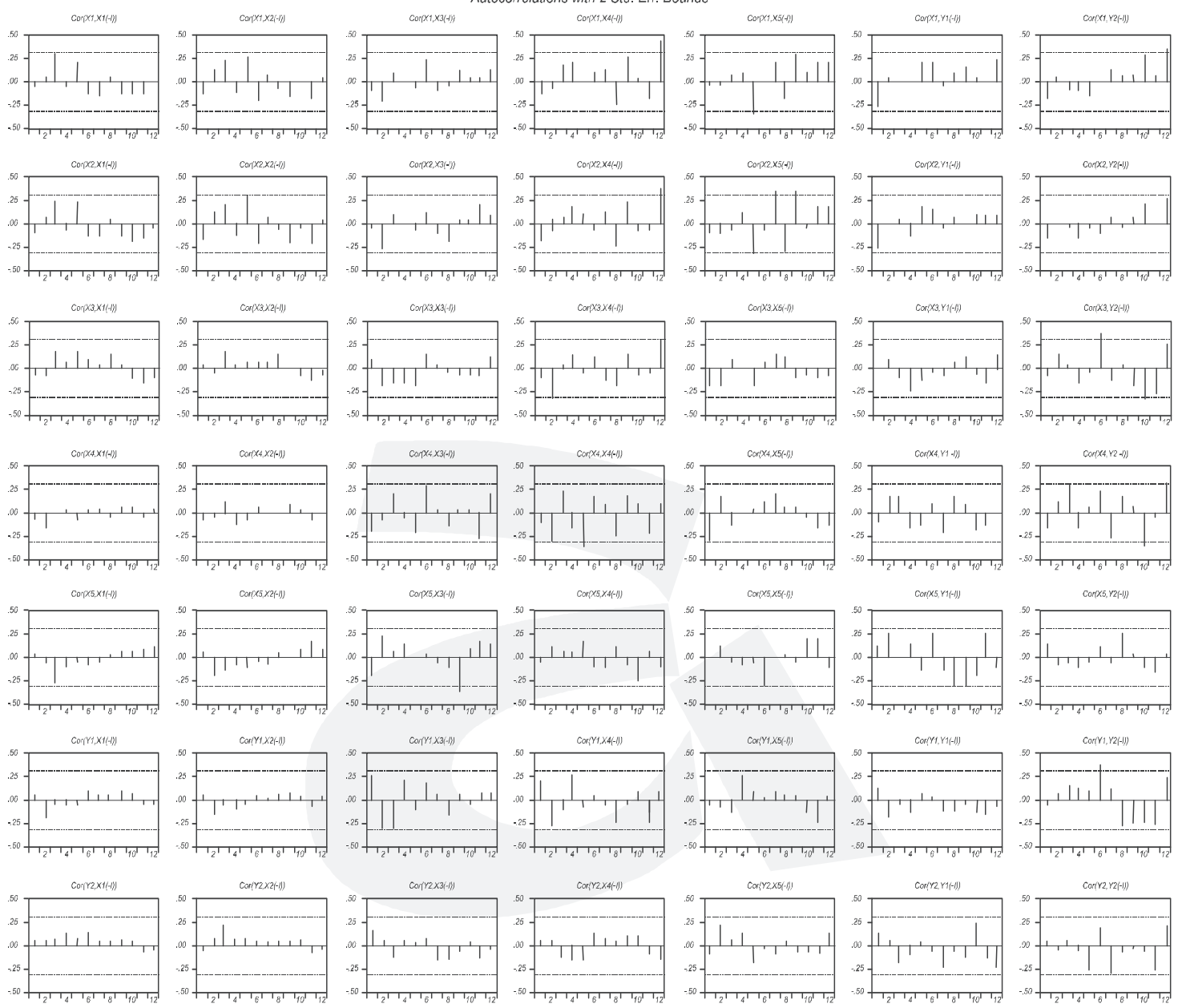

Source-Computed by author

The Doornik-Hansen Normality test showed that some component values of Chi-squares of skewness and kurtosis are insignificant and some components of Jarque Bera are also insignificant,therefore normality test is rejected at $5 \%$ level.In Table-3,the values are arranged. 
Table-3:Normality test

\begin{tabular}{|c|c|c|c|c|}
\hline Component & Skewness & Chi-square & $\mathrm{df}$ & Probability \\
\hline 1 & -1.02516 & 7.3394 & 1 & 0.0067 \\
\hline 2 & -0.34504 & 1.030359 & 1 & 0.3101 \\
\hline 3 & 0.000458 & $1.89 \mathrm{E}-06$ & 1 & 0.9989 \\
\hline 4 & -0.37256 & 1.194456 & 1 & 0.2744 \\
\hline 5 & 0.969224 & 6.701514 & 1 & 0.0096 \\
\hline 6 & -0.743477 & 4.278805 & 1 & 0.0386 \\
\hline 7 & 0.676342 & 3.621130 & 1 & 0.0571 \\
\hline Joint & & 24.16574 & 7 & 0.000 \\
\hline Component & kurtosis & Chi-square & $\mathrm{df}$ & Probability \\
\hline 1 & 5.462538 & 1.625122 & 1 & 0.2024 \\
\hline 2 & 5.194797 & 12.01844 & 1 & 0.0005 \\
\hline 3 & 4.744478 & 10.74461 & 1 & 0.0010 \\
\hline 4 & 4.830324 & 8.96958 & 1 & 0.0027 \\
\hline 5 & 4.980636 & 0.854511 & 1 & 0.3553 \\
\hline 6 & 3.544591 & 0.027912 & 1 & 0.8673 \\
\hline 7 & 4.384803 & 2.246676 & & 0.1339 \\
\hline Joint & & 34.48682 & 7 & 0.000 \\
\hline Component & Jarque Bera & $\mathrm{df}$ & Probability & \\
\hline 1 & 8.96495 & 2 & 0.0113 & \\
\hline 2 & 13.04880 & 2 & 0.0015 & \\
\hline 3 & 10.74461 & 2 & 0.0046 & \\
\hline 4 & 10.16400 & 2 & 0.0062 & \\
\hline 5 & 7.556024 & 2 & 0.0229 & \\
\hline 6 & 4.306717 & 2 & 0.1161 & \\
\hline 7 & 5.867806 & 2 & 0.0532 & \\
\hline Joint & 60.65256 & 14 & 0.000 & \\
\hline
\end{tabular}

Source-Computed by author

Granger Causality test showed that there are bi-directional causality among $\left(\mathrm{x}_{1}, \mathrm{x}_{2}\right),\left(\mathrm{x}_{1}, \mathrm{y}_{1}\right),\left(\mathrm{x}_{2}, \mathrm{x}_{3}\right),\left(\mathrm{x}_{2}, \mathrm{x}_{4}\right)$, $\left(x_{2}, x_{5}\right),\left(y_{1}, x_{2}\right),\left(x_{3}, x_{5}\right),\left(y_{1}, x_{3}\right),\left(y_{1}, x_{4}\right),\left(y_{1}, x_{5}\right)$ and there are uni-directional causality among $\left(\left(\mathrm{x}_{1}, \mathrm{x}_{3}\right),\left(\mathrm{x}_{1}, \mathrm{x}_{5}\right),\left(\mathrm{x}_{1}, \mathrm{x}_{4}\right),\left(\mathrm{x}_{3}, \mathrm{x}_{4}\right),\left(\mathrm{x}_{4}, \mathrm{x}_{5}\right)\right.$ respectively which are shown in the Table No-4 below. 
Table-4:Causality

\begin{tabular}{|l|l|l|l|}
\hline Null Hypothesis & Obs & F Statistic & probability \\
\hline$X_{2}$ does not Granger cause $x_{1}$ & 42 & 2.999 & 0.0912 \\
\hline$X_{1}$ does not Granger cause $x_{2}$ & & 0.01119 & 0.916 \\
\hline$X_{3}$ does not Granger cause $x_{1}$ & 42 & 8.153 & 0.0069 \\
\hline$X_{1}$ does not Granger cause $x_{3}$ & & 1.657 & 0.202 \\
\hline$X_{4}$ does not Granger cause $x_{1}$ & 42 & 24.318 & $2 \mathrm{E}-05$ \\
\hline$X_{1}$ does not Granger cause $x_{4}$ & & 17.0419 & 0.0002 \\
\hline$X_{5}$ does not Granger cause $x_{1}$ & 42 & 14.38 & 0.0005 \\
\hline$X_{1}$ does not Granger cause $x_{5}$ & & 2.02 & 0.1627 \\
\hline$Y_{1}$ does not Granger cause $x_{1}$ & 42 & 0.0136 & 0.907 \\
\hline$X_{1}$ does not Granger cause $y_{1}$ & & 0.372 & 0.545 \\
\hline$X_{3}$ does not Granger cause $x_{2}$ & 42 & 0.1847 & 0.6697 \\
\hline$X_{2}$ does not Granger cause $x_{3}$ & & 0.0041 & 0.9489 \\
\hline$X_{4}$ does not Granger cause $x_{2}$ & 42 & 2.199 & 0.1461 \\
\hline$X_{2}$ does not Granger cause $x_{4}$ & & 2.907 & 0.1368 \\
\hline$X_{5}$ does not Granger cause $x_{2}$ & 42 & 1.539 & 0.2221 \\
\hline$X_{2}$ does not Granger cause $x_{5}$ & & 1.324 & 0.2567 \\
\hline$Y_{1}$ does not Granger cause $x_{2}$ & 42 & 4.316 & 0.0444 \\
\hline$X_{2}$ does not Granger cause $y_{1}$ & & 2.0388 & 0.1613 \\
\hline$X_{4}$ does not Granger cause $x_{3}$ & 42 & 3.658 & 0.0631 \\
\hline$X_{3}$ does not Granger cause $x_{4}$ & & 2.2716 & 0.1398 \\
\hline$X_{5}$ does not Granger cause $x_{3}$ & 42 & 0.1985 & 0.6584 \\
\hline$X_{3}$ does not Granger cause $x_{5}$ & & 1.0764 & 0.3059 \\
\hline$Y_{1}$ does not Granger cause $x_{3}$ & 42 & 3.1819 & 0.0822 \\
\hline$X_{3}$ does not Granger cause $y_{1}$ & & 6.402 & 0.0155 \\
\hline$X_{5}$ does not Granger cause $x_{4}$ & 42 & 3.1819 & 0.0822 \\
\hline$X_{4}$ does not Granger cause $x_{5}$ & & 6.402 & 0.0155 \\
\hline$Y_{1}$ does not Granger cause $x_{4}$ & 42 & 0.4605 & 0.5014 \\
\hline$X_{4}$ does not Granger cause $y_{1}$ & & 0.9115 & 0.0 .345 \\
\hline$Y_{1}$ does not Granger cause $x_{5}$ & 42 & 0.1439 & 0.7064 \\
\hline$X_{5}$ does not Granger cause $y_{1}$ & & 0.6537 & 0.4237 \\
\hline & $40 u r c 0 m p u t e d ~ b y t h o r ~$ & & \\
\hline
\end{tabular}

Source-computed by author 


\section{VI.Limitation and future scope of research}

In this paper,the data of GDP and per capita GDP of India have been calculated from 18511883 on the basis of constant growth rates which were collected from Maddison(2006) to fit them into long term time series from 1851-1893.If the model is compared to other monetary systems in India or China,then a good comparative study would be achieved. However, there is ample scope for future research in this area of study.

\section{VII.Criticism}

The act of 1835 made silver rupee weighing 180 gms or $11 / 12$ th fine(containing $165 \mathrm{gms}$ of fine silver) in unlimited legal lender including demonetization of gold coins but act of 1841 introduced gold mohurs acceptance in public treasuries at the rate of $1: 15$. After 1850 production of silver tended to fall and currency famine intensified Indian economy.In 1861, paper currency Act passed and paper currency began to circulate.In 1874,government declared $£ 1=$ Rs10.5 and government adopted gold currency which led to fall price of silver from 58 pence to 37.5pence and to 27 pence in 1899 in which rupee sterling rate declined from 2 s(in 1872) to 1s2d in 1892 and India government faced a loss of 154 crores of rupees during 187589. In 1892, coinage of silver suspended and introduced $£ 1=R s 15$, or $R s 1=1 s 4 d$, and in 1893 the silver standard was abandoned.In Table- 5,it is shown that gold production rises with its rising price compared to silver including their index numbers during silver standard period from 1851 to 1892 which accelerated to demolish silver standard from India.

Table-5:Silver and gold production and price ratios.

\begin{tabular}{|l|l|l|l|l|}
\hline & $\begin{array}{l}\text { Ratio of gold } \\
\text { production to silver } \\
\text { production }\end{array}$ & $\begin{array}{l}\text { Ratio of value of } \\
\text { gold and silver }\end{array}$ & $\begin{array}{l}\text { Index no. of } \\
\text { production ratio }\end{array}$ & $\begin{array}{l}\text { Index no of } \\
\text { value of gold } \\
\text { and silver }\end{array}$ \\
\hline $1851-1855$ & 4.4 & 15.45 & 13.8 & 103.3 \\
\hline $1856-1860$ & 4.5 & 15.28 & 14.0 & 102.2 \\
\hline $1861-1865$ & 5.9 & 15.42 & 18.55 & 103.1 \\
\hline $1866-1870$ & 6.9 & 15.52 & 21.7 & 103.8 \\
\hline $1871-1875$ & 11.3 & 16.10 & 35.5 & 107.6 \\
\hline $1876-1880$ & 13.2 & 17.79 & 41.5 & 119.0 \\
\hline $1881-1885$ & 17.3 & 18.81 & 54.4 & 125.8 \\
\hline $1886-1890$ & 19.9 & 20.98 & 62.6 & 140.3 \\
\hline $1891-1895$ & 20.0 & 26.75 & 62.9 & 178.9 \\
\hline
\end{tabular}

Source-Ambedkar(1923)

Sir,R.Giffen remarked before the Fowler commission that India has abundant gold supply and in 1868, Sir R. Temple concludes to follow gold as legal tender.From 1870-1876, a fall of silver value prompted Bombay Chamber of Commerce recommended gold standard.Smith committee also proposed to follow gold standard.The spokesmen like Giffen, Mallet,
Farrer, Welby to Herschell committee (1893) were in favour of introduction of gold standard in India.

Viena congress(1859), Barlin congress (1863), Paris conference (1867) proclaimed stability, uniform coinage, gold as the principal currency. In 1871, Germany passed gold standard, in 
1872, Norway, Sweden, Denmark went to gold standard,in 1873 Belgium suspended silver standard, in 1879,Austria did the same, in 1873 USA passed gold standard and in 1878 Latin America suspended silver standard.During 1870-93 there was over supply of silver and silver price in terms of gold depreciated.Fisher also opposed to double standard with double coinages.England ultimately dominated world trade and finance in her favour adopting gold standard. But alas! India remains in gold exchange standard from 1893 onwards. Sterling was convertible to gold, rupee was convertible to sterling but rupee was not convertible to gold.

Capital inflows did not favour silver standard during 1851-1893 because one percent increases in gold inflows per year led to 0.65 decrease in GDP, $0.68 \%$ decrease in GDP per capita, $9.62 \%$ increase in export and $1.75 \%$ increase in import per year respectively which are all insignificant.On the other hand, one per cent rise in silver inflow per year led to $1.13 \%$ increase in GDP, $0.0012 \%$ decrease in GDP per capita, $13.45 \%$ increase in export and $12.03 \%$ increase in import per year respectively all of which are insignificant. Although, GDP ,export, import, gold silver price ratio had been increasing at the rates of $0.52 \%, 9.14 \%, 5.16 \%$ and $0.77 \%$ significantly per year respectively during the silver standard from 1851 to 1993. Therefore, instability of the silver standard in macroeconomic fundamentals is clear and the relations among them with capital flows were improper.

B.R.Ambedkar (1923) was dead against these outcomes of the silver standard in which the acts and laws were imposed as experiments during the silver standard by the British government although he fought for gold standard which was not introduced rather gold exchange standard was imposed in India from 1893.

\section{VIII.Conclusion}

The paper concludes that gold inflows during 1851-1893 had decreased at the rate of 0.34\% per year insignificantly but it was nonstationary,convergent and had no structural breaks. Silver inflows during 1851-1893 had increased at the rate of $1.51 \%$ per year insignificantly and found nonstationary and convergent and had one upward structural break in 1857. No co- integration among gold or silver inflows with GDP, GDP per capita,export,import and gold silver price ratio was found during 1851-1893 where VAR model was unstable and non-stationary and impulse response functions were diverging.Semi-log linear regression model among silver inflows and gold inflows with those variables were also insignificant although GDP,export, import and gold silver price ratio had been increasing at the rates of $0.52 \%$, $9.14 \%, 5.16 \%$ and $0.77 \%$ per year significantly. But double-log linear regression model suggested that gold inflows had significant impact from GDP,GDP per capita,export, and gold-silver price ratio but had no significant impact of silver inflows from those variables during 1851-1893 respectively. Yet,there is bidirectional causality among gold inflows,GDP, GDP per capita,export,import and gold silver price ratio significantly during the given period.Even,there were sharp depreciation of rupee sterling rate,silver price and production and gold price increased with production during the silver standard regime.Thus, gold and silver inflows could not synthesize the silver standard more effective in macro-dynamic adjustment during 1851-1893 although the series of managerial experiments of the commissions and government are equally responsible for instability of the silver standard in India which was equally identical with gold standard in England. 


\section{References}

Ambedkar,B.R.,1923, The problem of rupee:Its origin and solution,P.S.King \& sons Ltd, London.

Bai, Ying \& Kung, James Kai-sing., 2016, Opium, Silver and the Decline of Late Imperial China, 1789-1910, University of Hong Kong.

Bhowmik,Debesh.,2016,B.R.Ambedkar and Silver standard in India,paper will be presented in International seminar in Magadh University.

Bhowmik,Debesh.,1992-93,A profile of Dr.B.R.Ambedkar's view on the concept of Convertibility of Rupee,published in Indian Economic Association 76thConference volume1993-94,Bombay, 14-16, republished in in Debendra Kumar Das (ed)-Great Indian Economists:Their creative vision for socioeconomic development,314-320,(Deep and Deep Publications, NewDelhi)

Bhowmik,Debesh(ed).,2016, International Monetary System:Past,Present and Future(Regal Publishers, NewDelhi)

Bhowmik,Debesh.,2003,Essays on International Money,Deep and Deep Publishers, NewDelhi.

Bordo, Michael D.\& Meissner, Christopher M.,2007, Foreign Capital and Economic growth in the First Era of Globalisation,NBER.

Bordo, Michael David., 1981, Classical Gold Standard: Some Lessons Today, Federal Reserve Bank of St.Louis.

Ching, Jao Yu.,1965, The Silver Standard and China, 1911-1935, University of Hong Kong, Thesis for Master of Arts.

Coyajee,J.C., 1983, Development of Currency System in India, Deep and Deep Publications, NewDelhi

Das,Debendra Kumar.,2004, Relevance of Dr.Ambedkar's Economic Ideas on the Problems of Indian Currency:Some Observations, published in Indian Economic Association 76th Conference volume-1993-94, Bombay, 7-10, republished in
Debendra Kumar Das(ed)-Great Indian Economists: Their creative vision for socioeconomic development, 299-306, (Deep and Deep Publications, NewDelhi)

Davis,Lance E. \&Gallman, Robert E., 2001, Evolving Financial Markets and International Capital Flows:Britain, the Americas and Australia 1865-1914, Cambridge University Press.

Fernholz, Ricardo. Mitchener, Kris James \& Weidenenmier, Merc., 2013, Pulling up the Transished Anchor:The End of silver as a Global Unit of Account, www.cepr.org

Gault,John,2014, How does the gold price compare to other macro indicators? Chatham House Report Gold Taskforce Report. www.chathamhouse.org

Gonzalez,Rafael Dobado., Galvarriato, Aurora Gomez \& Williamson, J.G.,2008, Mexican exceptionalism:Globalisation and industrialization, Journal of Economic History, Vol-68,No-3,September.

Granger, C.W.J.,1969, Investigating Causal Relations by Econometric Models and Crossspectral Methods, Econometrica, 37(3), 424438.

Jadhav,Narendra.,2005, Exchange Rate Regime and Capital Flows:The Indian experience, Chief Economists' Workshop, April4-6, Bank of England.

Johansen,S., 1988,Statistical Analysis of Cointegrating Vectors. Journal of Economic Dynamics and Control, Vol. 12, 231-254.

................,1991, Estimation of Hypothesis Testing of Co-integration Vectors in Gaussian Vector Autoregressive Models, Econometrica 59,Nov, 1551-80

1994 , The role of the constant and linear terms in co-integration analysis of nonstationary variables. Econometric Reviews, 13,205-229 
Johansen, S., 1995, Likelihood-Based Inference in Co-integrated Vector Autoregressive

Models. Oxford University Press.

1996, Likelihood-Based Inference in Co-integrated Vector Autoregressive Models, 2ndEdition,Oxford University Press.

\& K.Juselius.,1990,Maximum likelihood estimation and co-integration with application to the demand of money. Oxford Bulletin of Economics and Statistics,Vol-52,No-2,169-210.

Keynes, J.M., 1913, Indian currency and finance". Macmillan and co.

Leavens, Dickson.H.,1939,Silver Money,Principia Press Inc,Bloomington, Indiana

Ma,Debin.,2012,Money and Monetary System in China in the 19-20th century:An Overview, Working Paper no-159/12,London School of Economics.

Maddison, A., 2006, The World Economy: Vol-1 and 2. OECD: Paris

,2007, Contours of the world economy,1-2030:Essays in Macro Economic History,OUP.
O'Connor,F.A. et al.,2015, The Financial Economics of Gold -A Survey, International Review of Financial Analysis,41,186-205.

Roy, Alokananda.,2004,Ambedkar versus Keynes-On Indian Currency:In the Light of Contemporary Relevance,published in published in Indian Economic Association 76thConference volume-1993-94,Bombay,11-13 republished in Debendra Kumar Das (ed)-Great Indian Economists:Their creative vision for socioeconomic development,307-313,(Deep and Deep Publications,NewDelhi)

SRSrocco Report.,2015,Filed in Energy,Precious metals, Dec6, https://srsroccoreport.com

Velde,Francois R.\& Weber, Warren E.,1998,A Model of Bimetallism,Federal Reserve Bank of Minneapolis, Research Department, Working Paper-588.

World Gold Council,2010,The 10yr gold bull market in perspective, London,UK.

Zaraga,Ludovico.,2014,Effects of Exchange Rate Regimes on Trade: the Case of Silver Standard India,Northwestern University,Nov, 24. 